\title{
Is there a relationship between screening for diabetes related foot complications and limb amputation?
}

\author{
Ainslie Davies ${ }^{1,2^{*}}$, Peter Lazzarini ${ }^{1,2,3}$, Bishnu Sharma ${ }^{4}$, Lloyd Reed ${ }^{1,2}$ \\ From Australasian Podiatry Conference 2015 \\ Queensland, Australia. 6-8 May 2015
}

\section{Background}

From the conservative estimates of registrants with the National Diabetes Supply Scheme, we will be soon passing 1.1 Million Australians affected by all types of diabetes. The diabetes complications of foot ulceration and amputation are costly to all. These costs can be reduced with appropriate prevention strategies, starting with identifying people at risk through primary care diabetic foot screening. Yet levels of diabetic foot screening in Australia are difficult to quantify. This presentation aims to report on foot screening rates as recorded in existing academic literature, national health surveys and national database reports.

\section{Methods}

Literature searches included diabetic foot screening that occurred in the primary care setting for populations over 2000 people from 2002 to 2014. Searches were performed using Medline and CINAHL as well as internet searches of Organisations for Economic Co-operation and Development (OECD) countries health databases. The focus is on type 1 and type 2 diabetes in adults, and not gestational diabetes or children. The two primary outcome measures were foot-screening rates as a percentage of adult diabetic population and major lower limb amputation incidence rates from standardised OECD data.

\section{Results}

The most recent and accurate level for Australian population review was in the AUSDIAB (Australian Diabetes

\footnotetext{
* Correspondence: ainslie.davies@qut.edu.au

'School of Clinical Sciences, Queensland University of Technology, Brisbane, Qld, 4590, Australia

Full list of author information is available at the end of the article
}

and lifestyle survey) from 2004. This survey reported screening in primary care to be as low as $50 \%$. Countries such as the United Kingdom and United States of America have much higher reported rates of foot screening (67-86\%) recorded using national databases and web based initiatives that involve patients and clinicians. By comparison major amputation rates for Australia were similar to the United Kingdom at 6.5 versus 5.1 per 100,000 population, but dis-similar to the United States of America at 17 per 100,000 population.

\section{Conclusions}

Australian rates of diabetic foot screening in primary care centres is ambiguous. There is no direct relationship between foot screening levels in a primary care environment and major lower limb amputation, based on national health survey's and OECD data. Uptake of national registers, incentives and web-based systems improve levels of diabetic foot assessment, which are the first steps to a healthier diabetic population.

\footnotetext{
Authors' details

'School of Clinical Sciences, Queensland University of Technology, Brisbane, Qld, 4590, Australia. ${ }^{2}$ Institute of Health and Biomedical Innovation, Queensland University of Technology, Brisbane, Queensland, Australia. ${ }^{3}$ Allied Health Research Collaborative, Metro North Hospital \& Health Service, Brisbane, Queensland, Australia. ${ }^{4}$ School of Business, University of the Sunshine Coast, Qld, 4556, Australia.

Published: 22 September 2015

doi:10.1186/1757-1146-8-S2-011

Cite this article as: Davies et al:: Is there a relationship between

screening for diabetes related foot complications and limb amputation? Journal of Foot and Ankle Research 2015 8(Suppl 2):011.
} 